\title{
A New Bridge Functional and Its Application to Density Func- tional Approach for Non-uniform Fluid*
}

\author{
Zhou Shi-Qi Zhang Xiao-Qi \\ ( Research Institute of Modern Statistical Mechanics, Zhuzhou Institute of Technology, Zhuzhou 412008)
}

\begin{abstract}
To extend the recently proposed density functional theory (DFT) methodology to non-uniform non-hard sphere fluid, a new bridge functional as a function of indirect correlation function was proposed, which was based on analysis on the asymptotic behavior of the Ornstein-Zernike equation system and the Taylor expansion series whose re-normalization resulted in an adjustable parameter determined by combining the new bridge functional with the DFT methodology to make the contact density from a single hard wall satisfy the sum rule. The combination of the new bridge functional and the DFT methodology predicted well the density distribution profile of hard sphere fluid near a hard wall or confined in a spherical cavity, Lennard-Jones fluid and adhesive hard sphere fluid confined between two hard walls when compared with the available computer simulation data.
\end{abstract}

Keywords: Density functional theory, Bridge functional, Adhesive hard sphere fluid, Integral equation theory, Hard sphere fluid

A completely new density functional theory (DFT) formalism ${ }^{[1]}$ was recently proposed by the present author which resulted from the use of the universality concept of the free energy density functional and the test particle trick. The new DFT formalism was extended ${ }^{[2-8]}$, the universality principle and the test particle trick were employed in a different manne by the following papers ${ }^{[0-11]}$. The new formalism differs from the previous three categories of the DFTs, i.e. the functional perturbative expansion approximation(PEA), the weighted density approximation (WDA) and its various variants, and the fundamental measure theory ${ }^{[12-14]}$.

The new methodology used as input the bulk bridge functional which is required to be expressed as a function of indirect correlation function $\gamma$, such kinds of bridge functional include the PY bridge functional ${ }^{[15]}$, verlet-modified(VM) bridge function- al, Martynov-Sarkisov(MS) bridge functional ${ }^{[15]}$. But all of these bridge functional forms is only suitable for hard sphere fluid. To apply the methodology to non-uniform non-hard sphere fluid, mixed bridge functional had to be employed ${ }^{[2]}$. To go beyond the bridge functional concept, the universality principle and the test particle trick were combined to incorporate the bulk radial distribution function into the approximation to make very good predictions for the density distribution profile ${ }^{[9-11]}$, but these reformulated formalisms were a little more computationally complicated. The present contribution was still based on the bridge functional concept, but new analytical bridge functional was proposed.

\section{The new bridge functional}

With reference to the previous bridge functionals, for example, the PY approximation, the VM

Received: December 26, 2001; Revised: March 1, 2002. Correspondent: Zhou Shi-Qi(E-mail: chixiayzsq@ yahoo. com). ${ }^{*}$ The Project 
approximation, the MS approximation, etc., we denote the bridge functional as a function of indirect correlation function $\gamma$ by $B(\gamma)$, expanding $B(\gamma)$ around $\gamma=0$

$$
\begin{aligned}
B(\gamma) & =B(0)+B^{\prime}(0) \gamma+\frac{B^{\prime \prime}(6)}{2 !} \gamma^{2}+\frac{B^{\prime \prime \prime}(0)}{3 !} \gamma^{3} \\
& +\frac{B^{(4)}(0)}{4 !} \gamma^{4}+\cdots+\frac{B^{(6)}(0)}{n !} \gamma^{n}+\cdots
\end{aligned}
$$

the bulk Ornstein-Zernike equation ${ }^{[15]}$ reads

$$
\begin{gathered}
\gamma=h(r)-C_{0}^{(2)}\left(r ; \rho_{\mathrm{b}} \cdots\right)= \\
\rho_{\mathrm{b}} \int \mathrm{d} r^{\prime} h\left(r^{\prime}\right) C_{0}^{(2)}\left(r, r^{\prime} ; \rho_{\mathrm{b}} \cdots\right)
\end{gathered}
$$

the closure equation reads

$$
g(r)=\exp [-\beta u(r)+\gamma(r)+B(\gamma)]
$$

where, $h(r)=g(r)-1$, is the bulk total correlation function, $g(r)$ is the bulk radial distribution function, $\beta u(r)$ is the inter-particles interaction potential and $C_{0}^{(2)}\left(r, r^{\prime}, \rho_{\mathrm{b}} \cdots\right)$ is the bulk second order direct correlation function(DCF) in which $\left(\rho_{\mathrm{b}} \cdots\right)$ stands for the bulk parameters. When the inter-particles separation is very large, $\beta u(r) \rightarrow 0, h(r) \rightarrow 0$, from Eq. (2) we know $\gamma(r) \rightarrow 0$ as $r \longrightarrow \propto$, finally from Eq.

(3) we know that $B(\gamma) \rightarrow 0$ as $\gamma(r) \rightarrow 0$ which leads to $B(0)=0$ when combined with Eq. (1).

To ascertain the other coefficients in the expansion Eq. (1), we compare the Eq. (1) with the expansions of other approximate bridge functionals ${ }^{[15]}$. For example, the PY bridge functional

$$
B^{\mathrm{PY}}(\gamma)=\ln (1+\gamma)-\gamma
$$

the MS bridge functional

$$
B^{\mathrm{MS}}(\gamma)=(1+2 \gamma)^{1 / 2}-\gamma-1
$$

the VM bridge functional

$$
B^{\mathrm{VM}}(\gamma)=\frac{-\gamma^{2}}{2(1+4 \gamma / 5)}
$$

Now we expand the Eqs. $(4 \sim 6)$ around $\gamma=0$,

$$
\begin{aligned}
& B^{\mathrm{PY}}(\gamma)=-0.5 \gamma^{2}+0.3333 \gamma^{3} \mp \cdots \\
& B^{\mathrm{MS}}(\gamma)=-0.5 \gamma^{2}+0.5 \gamma^{3} \mp \cdots \\
& B^{\mathrm{VM}}(\gamma)=-0.5 \gamma^{2}+0.4 \gamma^{3} \mp \cdots
\end{aligned}
$$

From Eqs. $(7 \sim 9)$, we know that it is reasonable to make $B^{\prime}(0)=0.0$ and $\frac{B^{\prime \prime}(0)}{2 !}=-0.5$, thus Eq. (1) reduces to

$$
\begin{aligned}
& B(\gamma)=-0.5 \gamma^{2}+\frac{B^{\prime \prime \prime}(6)}{3 !} \gamma^{3}+\frac{B^{(4)}(0)}{4 !} \gamma^{4}+\cdots \\
& +\frac{B^{(n)}(0)}{n !} \gamma^{\mathrm{n}}+\cdots \\
& =-0.5 \gamma^{2}\left(1-\frac{2 B^{\prime \prime \prime}(0)}{3 !} \gamma-\frac{2 B^{(4)}(0)}{4 !} \gamma^{2}-\right. \\
& \left.\cdots-\frac{2 B^{(6)}(0)}{n !} \gamma^{n-2}-\cdots\right) \\
& =-0.5 \gamma^{2}\left(1-\frac{2 B^{\prime \prime \prime}(0) / 3 !}{1 !} \gamma-\frac{2 B^{4)}(6) 2 ! / 4 !}{2 !} \gamma^{2}\right. \\
& \left.\quad-\cdots-\frac{2 B^{(6)}(0)(n-2) ! / n !}{(n-2) !} \gamma^{n-2}-\cdots\right)
\end{aligned}
$$

The series in Eq. (10) could not be truncated because $\gamma$ is not a very small number. It is desirous to approximate the series in Eq. (10) by a function, considering the similarity between the above series and the expansion of exponential function. It is reasonable for one to approximate the series in Eq. (10) with the exponential function $\exp (-\alpha \gamma)$, thus

$$
B(\gamma)=-0.5 \gamma^{2} \exp (-\alpha \gamma)
$$

Eq. (11) is the main result of the present contribution. However it should be noted that the exponential function $\exp (-\alpha \gamma)$ with arbitrary $\alpha$ is not consistent with the series in Eq. (10) with a fixed function form $B(\gamma)$ because each of the terms in the series in Eq. (10) is not equal to the corresponding term of the expansion of $\exp (-\alpha \gamma)$, but the substitution avoids the truncation of the series, by choosing the appropriate parameter $\alpha$, the approximation on each term of the series in Eq. (10) is smoothed out and canceled out globally, thus the final error can be expected to be reduced. So one can call this process as re-normalization.

In the formalism of DFT, the density profile equation of a non-uniform single component fluid is

$$
\begin{aligned}
\rho(r)= & \rho_{\mathrm{b}} \exp \left\{-\beta \varphi_{\mathrm{ext}}(r)+C^{(1)}(r ;[\rho])-\right. \\
& \left.C_{0}^{(1)}\left(\rho_{\mathrm{b}}\right)\right\}
\end{aligned}
$$

where $\varphi_{\text {ext }}(r)$ is the external potential responsible for the generation of the density distribution $\rho(r), \beta=$ $1 / k T$ with $k$ the Boltzmann constant and $T$ the absolute temperature, $C^{(1)}$ and $C_{0}^{(1)}$ are the first order direct correlation function and its uniform counterpart.

According to the methodology in Ref [1] and the 
present bridge functional Eq. (11), Eq. (12) reduces to

$$
\begin{aligned}
& \rho(r)=\rho_{\mathrm{b}} \exp \left\{-\beta \varphi_{\mathrm{ext}}(r)+\int \mathrm{d} r^{\prime}\left(\rho\left(r^{\prime}\right)-\rho_{\mathrm{b}}\right) \cdot\right. \\
& C_{0}^{(2)}\left(r, r^{\prime} ; \rho_{\mathrm{b}} \cdots\right)+ \\
& \left.B\left(\int \mathrm{d} r^{\prime}\left(\rho\left(r^{\prime}\right)-\rho_{\mathrm{b}}\right) C_{0}^{(2)}\left(r, r^{\prime} ; \rho_{\mathrm{b}} \cdots\right)\right)\right\}
\end{aligned}
$$

Then the numerical value of $\alpha$ was determined by the sum rule which specifies the bulk pressure $p$ by the hard wall contact density $\rho_{\mathrm{w}}$

$$
p=\rho_{\mathrm{w}} k T
$$

$\rho_{\mathrm{w}}$ can be obtained from $\rho(0.5)$ in Eq. (13) when the external potential has the following form

$$
\begin{aligned}
\varphi_{\mathrm{ext}}(z) & \rightarrow \propto \quad z / \sigma<0.5 \\
& \rightarrow 0 \quad z / \sigma>0.5
\end{aligned}
$$

Although the specification of the adjustable parameter $\alpha$ is dependent on the hard wall contact density, this does not limit the present approach to the application to a fluid in a single hard wall confinement, this can be explained as follows. Obviously the numerical value of $\alpha$ is related to $C^{(1)}(r$; $[\rho])-C_{0}^{(1)}\left(\rho_{\mathrm{b}}\right)$ from Eq. (12) and (13), $C^{(1)}(r$; $[\rho])$ is the functional derivative of the excess Helmholtz free energy density functional $F_{\text {ex }}[\rho]$ with respective to the density distribution.

$$
C^{(1)}(x ;[\rho])=-\beta \frac{\delta F_{\text {ex }}[\rho]}{\delta \rho(r)}
$$

The universality of the $F_{\mathrm{ex}}[\rho]^{[1,}{ }^{16]}$ means its independence on the concrete external potential responsible for the formation of the density distribution profile. From the relationship Eq. (16), $C^{(1]}(r,[\rho])$ $-C_{0}^{(1)}\left(\rho_{\mathrm{b}}\right)$ is also universal, thus the adjustable parameter $\alpha$ is also universal and not dependent on the external potential, i. e., it is only related to the bulk parameter $\rho_{\mathrm{b}} \cdots$. Thus, so specified $\alpha$ in this special case (a single hard wall) can be used for an arbitrary external potential case!

As an example, we consider a non-uniform hard sphere fluid near a single hard wall for which the external potential is of the form of Eq. (15) . For hard sphere fluid the second order DCF $C_{0}^{(2)}\left(r ; \rho_{\mathrm{b}} \cdots\right)$ from the Percus-Yevick approximation ${ }^{[17-18]}$ was em- ployed in Eq. (13). The equation of the state for the hard sphere fluid was from the Carnanhan-Startling empirical one ${ }^{[19]}$. In Fig. 1, we plotted the density distribution profile and the corresponding computer simulation data ${ }^{[20]}$ for the case of the external potential Eq. (15) at two bulk density, also displayed was the prediction from the VM bridge functional which was shown to be the most accurate predictions for non-uniform hard sphere fluid in Ref[1]. From Fig. 1, one can see that the present predictions are even more accurate than that based on the VM bridge functional.

To indicate that the present method was not limited to a fluid near a single hard wall and also can be employed for the other external potential cases, we employed the present method to calculate the density distribution profile for a hard sphere fluid confined in a spherical cavity whose external potential is of the following form

$$
\begin{aligned}
\varphi_{\text {ext }}(r) & \rightarrow \propto & |r| / \sigma>R \\
\longrightarrow 0 & & |r| / \sigma<R
\end{aligned}
$$

As mentioned above, the adjustable parameter for the case Eq. (17) can be obtained from the case of the same bulk fluid near a single hard wall. The calculated density distribution was plotted against the corresponding simulation data ${ }^{[21]}$ in Fig. 2 for two bulk densities. The agreement with the simulation

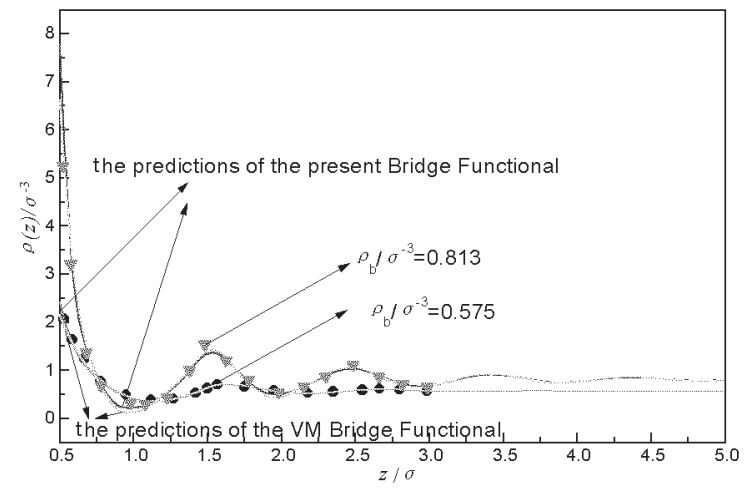

Fig. 1 Density profiles of a hard sphere fluid near a hard wall for two cases of bulk densities The lines correspond to the predictions of the theories, the symbols stand for the corresponding computer simulation data ${ }^{[20]}$. 


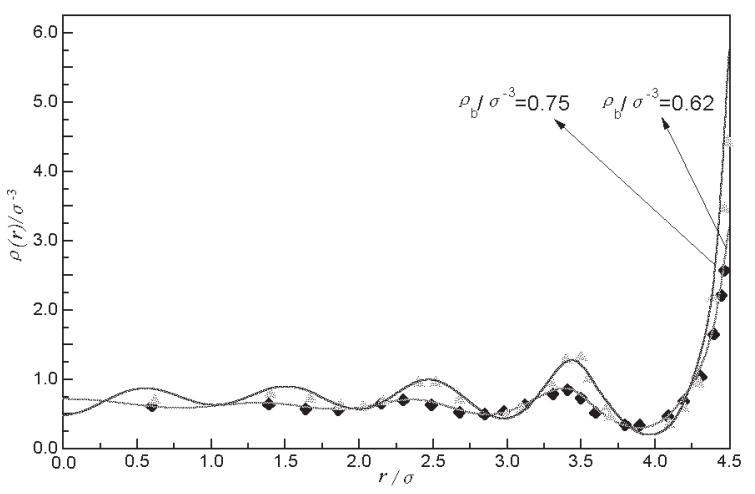

Fig. 2 Density profiles of a hard sphere fluid confined in a spherical cavity with a hard wall $R=4.5 \sigma$ for two cases of bulk densities

The lines correspond to the predictions of the present method, the symbols stand for the corresponding computer simulation data ${ }^{[21]}$.

data was also good. This clearly indicated the independence of the adjustable parameter $\alpha$ on the external potential.

To see whether the present bridge functional was applicable to the non-hard sphere fluid, we applied it to the Lennard-Jones fluid confined between two hard walls at separation $H$. For this case the external potential has the following form

$$
\begin{aligned}
\varphi_{\text {ext }}(z) & \rightarrow \propto \quad z / \sigma<0.5 \text { or } z / \sigma>H-0.5 \\
& \rightarrow 0 \quad 0.5<z / \sigma<H-0.5
\end{aligned}
$$

For LJ fluid the interaction potential has the following form

$$
u(r)=4 \varepsilon\left[(\sigma / r)^{12}-(\sigma / r)^{6}\right]
$$

where $\sigma$ and $\varepsilon$ characterize the interaction range and strength, respectively.

To proceed computationally, the quantity $C_{0}^{(2)}$ $\left(r, r^{\prime} ; \rho_{\mathrm{b}} \cdots\right)$ was obtained by solving numerically the $\mathrm{OZ}$ equation with the bridge functional from Ref [22] for uniform $\mathrm{LJ}$ fluid employed

$$
B(r)=-s^{2} / 2[1+s(5 s+11) /(7 s+9)]
$$
where $s=h(r)-C_{0}^{(2)}\left(r ; \rho_{\mathrm{b}}\right)-\beta u_{2}(r)$ and $u_{2}(r)=$ $-4 \varepsilon(\sigma / r)^{6} \exp \left[\left(-1 / \rho^{x}\right)(\sigma / r){ }^{6 \sigma^{*}}\right]$

with $\rho^{x}=\rho_{\mathrm{b}} \sigma^{3}$. The bulk pressure $p$ can be obtained from an equation of state for bulk LJ fluid ${ }^{[23]}$.

It should be noted that we did not employ the present bridge functional to solve the $\mathrm{OZ}$ equation, because the present bridge functional was devised to be used in the DFT. Now one did not know whether the present bridge functional can produce good predictions for the bulk second order DCF $C_{0}^{(2)}\left(r ; \rho_{\mathrm{b}} \cdots\right)$ which was the key input into the DFT, but we knew that the bridge functional of Eq. (20) can produce good predictions for this quantity.

The density profile equation(13) for the above non-uniform LJ fluid was plotted and compared with the corresponding computer simulation results ${ }^{[24]}$ in Fig. 3 for $H / \sigma=5$ and 13. Also the predictions from the second order perturbation DFT were plotted in the same figures. From Fig. 3 one can see that the predictions of the present method are far more superior to that from the second order perturbation DFT and are in good agreement with the corresponding computer simulation results.

Now we consider an adhesive hard sphere fluid confined between two hard walls situated at $z=-$ 0. 5. $R$ and $z=H+0.5 R$ respectively, for this case the external potential is given by

$$
\beta \varphi_{\text {ext }}(z) \rightarrow \begin{cases}0 & 0<z<H \\ \propto & \text { otherwise }\end{cases}
$$

The interaction potential ${ }^{[24]}$ of the adhesive hard sphere fluid has the following form

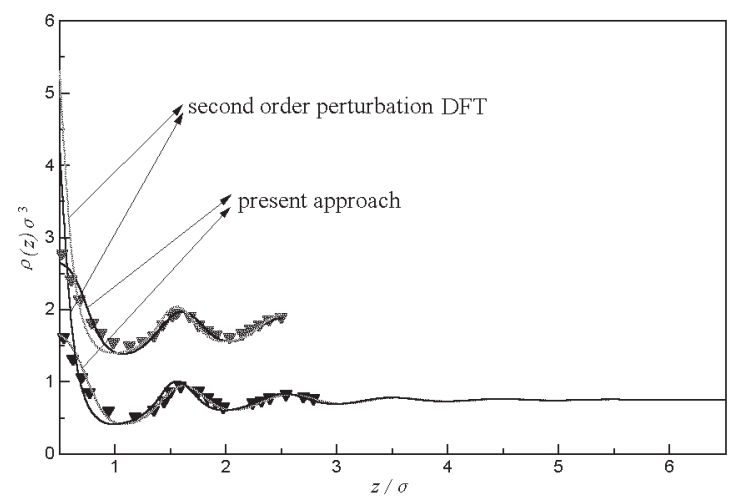

Fig. 3 Density profiles of a Lennard-Jones fluid $\left(\rho_{\mathrm{b}} \sigma^{3}=0.75, k T / \varepsilon=1.304\right) \quad$ confined between two hard walls with $H / \sigma=5$ (the above curve) and $H / \sigma=13$ (the below curve) respectively The dots represent the corresponding MC results ${ }^{[24]}$. Only half the slit is shown. The above curve should be shifted down a unit. 


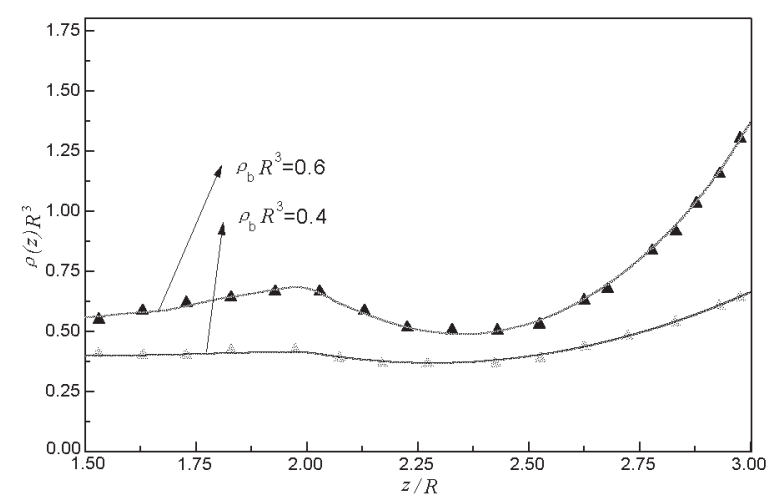

Fig. $4 \quad$ Density profile $\rho(z) R^{3}$ vs $z / R$ for an adhesive hard sphere fluid confined between two hard walls with wall separation of $3 R$ at two different bulk densities $\rho_{\mathrm{b}} \boldsymbol{R}^{3}$

The stickiness parameter is $\tau=0.5$, the curves stand for the predictions from the present bridge functional, the dots represent the corresponding computer simulation data ${ }^{[28]}$.

$$
\begin{array}{rrr}
\beta \phi(r) \rightarrow \propto, & 0<r<R^{\prime} \\
=-\ln \left(\frac{R}{12 \tau\left(R-R^{\prime}\right)}\right), & R^{\prime}<r \leqslant R \\
\rightarrow 0, & r>R
\end{array}
$$

where $\tau$ is a dimensionless parameter which increases with increasing temperature, and $\tau^{-1}$ is a parameter representing a measure of the adhesiveness of the potential.

To proceed computationally, $C_{0}^{(2)}\left(r, r^{\prime} ; \rho_{\mathrm{b}} \cdots\right)$ of the adhesive hard sphere fluid was employed from the PY approximation

$$
\begin{gathered}
C_{0 \text { adh }}^{(2)}\left(r, \rho_{\mathrm{b}}, \tau\right)=(-a-b r / R- \\
\left.\frac{1}{2} \eta a(r / R)^{3}-\frac{1}{12} \eta \lambda^{2} R / r\right) \cdot \\
\Theta(R-r)+\frac{1}{12} \lambda \delta\left(r-R^{-}\right)
\end{gathered}
$$

where $a, \quad b$ or $\lambda$ is a function of $\eta=\rho_{\mathrm{b}} \sigma^{3} \pi / 6$ and $\tau^{[25]}$.

The bulk pressure $p$ for the adhesive hard sphere fluid can be obtained from the equation of state which is via the compressibility equation ${ }^{[26-27]}$.

$$
\frac{\beta p}{\rho_{\mathrm{b}}}=\frac{1+\eta+\eta^{2}}{(1-\eta)^{3}}-\frac{\xi(2+\eta)}{2(1-\eta)^{3}}+\frac{\xi^{3}}{36 \eta(1-\eta)^{3}}
$$

The predictions from Eq. (13) for the above non-uniform adhesive hard sphere fluid were plotted with the corresponding computer simulation data ${ }^{[28]}$ in

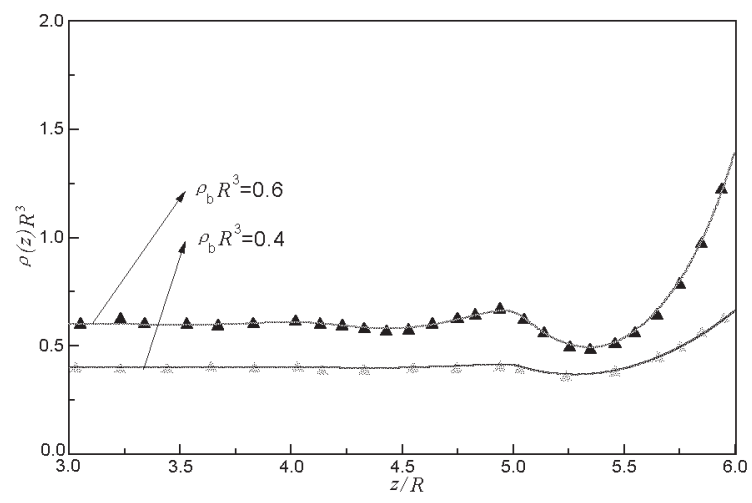

Fig. 5 The same as in Fig. 4., but with wall separation of $6 R$

Figs. 4, 5 .

For the calculated cases, it was shown that the predictions from the present bridge functional described well the oscillatory density profiles with period of oscillation equal to the particle diameter which was indicative of the formation of the molecular layers, the agreement with the simulation data was very good. Also one can see that the contact density for the adhesive hard sphere fluid was largely lowered compared with that of the hard sphere fluid because of the adhesiveness between the particles forcing the particles to move away from the walls to have more chance for the energetically favorable sticking between each other.

\section{Concluding remarks}

The present bridge functional includes three approximations, one was to regard the bridge function as a functional of the indirect correlation function, the second was to approximate the series in Eq. (10) by an exponential function $\exp (-\alpha \gamma)$, finally the third one was to make $B^{\prime}(0)=0.0$ and $\frac{B^{\prime \prime}(6)}{2 !}=-0.5$ with reference to some previous bridge functionals. The improvement over the present bridge functional was thus to incorporate more adjustable parameters and employed the so-called zero-separation theorems ${ }^{[29-31]}$ to specify $B^{\prime}(0)$ and $\frac{B^{\prime \prime}(0)}{2 !}$, or to re-normalize the indirect correlation function ${ }^{[29]}$ for non-hard 
sphere interaction potential fluid. It should be noted that another important application of the present bridge functional was to use it to close the $\mathrm{OZ}$ equation to predict thermodynamic and structure properties of bulk fluid. We will report the improvement and detailed application of the new bridge functional Eq. (11) to the above mentioned field in some separate papers.

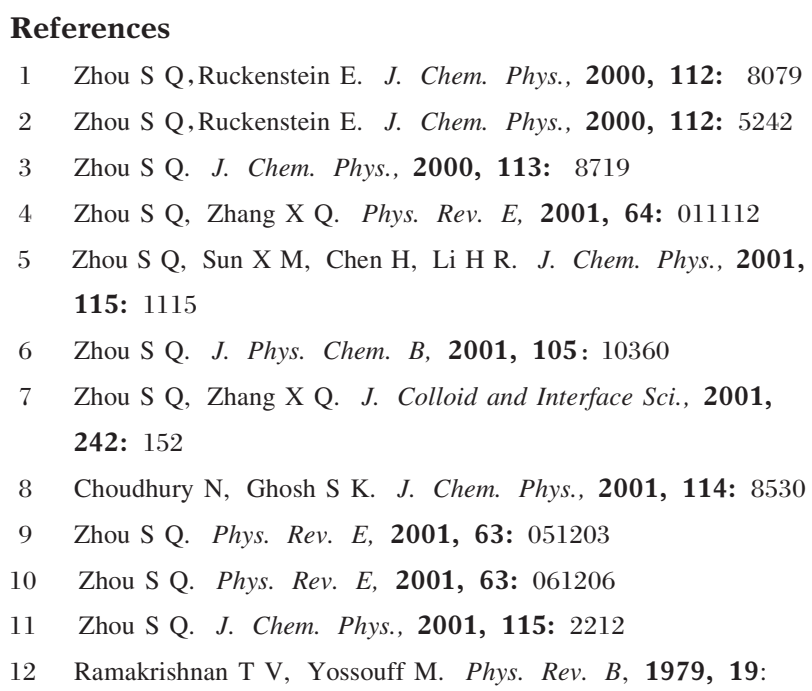

2775

13 Tarazona P. Phys. Rev. A, 1985, 31: 2672

14 Rosenfeld Y. Phys. Rev. Lett., 1989, 63: 980

15 Hansen J P, McDonald I R. Theory of Simple Liquids. 2nd ed. New York: Academic, 1986

16 Chayes J T, Chayes L. Commun. Math. Phys., 1984, 93: 57

17 Thiele E. J. Chem. Phys., 1963, 39: 474

18 Wertheim M S. Phys. Rev. Lett., 1963, 19: 321

19 Carnahan N F, Starling K E. J. Chem. Phys., 1969, 51: 635

20 Groot R D, Faber N M, van der Eerden J P. Mol. Phys., 1987, 62: 861

21 Calleja M, North A N, Powels J G, Rickayzen G. Mol. Phys. 1991, 73: 973 Johnson J K, Zollweg J A, Gubbins K E. Mol. Phys., 1993, 78: 591

24 Powles J G, Rickayzen G, Williams M L. Mol. Phys., 1988, 64: 33

25 Baxter R J. J. Chem. Phys., 1968, 49: 2770

26 Tejero C F, Baus M. Phys. Rev. E, 1993, 48: 3793

27 Wang J, McLaughlin L, Silbert M. Mol. Phys., 1993, 79: 704

28 Jamnik A, Bratko D. Phys. Rev. E, 1994, 50: 1151

29 Meeron E, Siegert A J F. J. Chem. Phys., 1968, 48: 3139

30 Grundke E W, Henderson D. Mol. Phys., 1972, 24: 269

31 Lee L L. J. Chem. Phys., 1995, 103: 9388

32 Bomont J M, Bretonnet J L. J. Chem. Phys., 2001, 114: 4141

\title{
一个新的桥泛函及其在非均一流体密度泛函理论中的应用 *
}

\author{
周世琦张晓祺 \\ (株洲工学院现代统计力学研究所, 株洲 412008)
}

摘要 基于对 OZ 方程的渐近行为与 Taylor 级数展开的分析, 提出了一个新的桥泛函, 桥泛函被表达为间 接相关函数的函数, Taylor 级数展开的重整化导致了一个可调参数, 通过将所提出的桥泛函与一个最近提出 的密度泛函理论方法学, 以及单个硬墙的 sum 规则结合, 可以确定可调参数. 所提出的桥泛函能预言如下 非均一流体的密度分布: 硬球流体接近一个硬墙与在球形空隙内, Lennard-Jones 流体与缔合硬球流体在两 个硬墙之内．理论预言与文献所报导的模拟数据符合很好.

关键词：密度泛函理论，桥泛函，缔合硬球流体，积分方程理论，硬球流体 中图分类号: 0641 